\title{
Pressure-driven filling of liquid metal in closed-end microchannels
}

\author{
Alfonso M Gañán-Calvo, ${ }^{1, *}$ Wei Guo,${ }^{2,3}$ Heng-Dong Xi, ${ }^{2}$ Adrian J.T. Teo, ${ }^{3}$ Nam-Trung Nguyen, ${ }^{3}$ and Say Hwa Tan ${ }^{3}, \dagger$ \\ ${ }^{1}$ Depto. de Ingeniería Aeroespacial y Mecánica de Fluidos, Universidad de Sevilla, E-41092 Sevilla, Spain. \\ ${ }^{2}$ School of Aeronautics, Northwestern Polytechnical University, 127 West Youyi Rd, Xi'an, Shaanxi, China. \\ ${ }^{3}$ Queensland Micro and Nanotechnology Centre, Griffith University, 170 Kessels Road, QlD, 4111, Australia.
}

(Dated: May 31, 2019)

\begin{abstract}
We observed an unsteady flow behaviour of liquid metal during a pressure-driven injection process into a closed-ended PDMS microchannel. A constant pressure is applied at the inlet to allow Eutectic Gallium-Indium (EGaIn) to completely fill the porous microchannels. In contrast to open channels (Dickey et al. 2008, Adv. Functional Mater. 18, 1097), the flow exhibits a complex unsteady behavior with sudden random length jumps and time stops. However, with appropriate formulating of a suitable mathematical model with the system using (i) the permeability of PDMS to air, (ii) previous descriptions of the nature of EGaIn surface oxide layer (SOL), and (iii) a key probabilistic approach, we show that the average quantities defining the quantum-like flow can be accurately predicted. The proposed probabilistic formulation provides for the first time; a description of the dynamics of the SOL, the breaking and healing characteristic times when EGaIn is driven in a microchannel. Importantly, this work provides a better understanding on the complex flow behavior and lays the foundation for future works.
\end{abstract}

\section{INTRODUCTION}

Fluid displacement in porous media is of great importance to many diverse fields of science and engineering such as petroleum displacement in rocks [1], resin transfer molding of composite material production [2] and manipulation of liquid metals in microelectromechanical (MEMS) and microfluidic systems [3]. The manipulation of liquid metals has great potentials for creating components in MEMS and microfluidic systems, such as switches [4], pumps [5], sensors [6], electrodes [7], and reflectors [8]. This is due to the fact that liquid metal is in liquid state at room temperatures. Gallium alloys such as Eutectic Gallium-Indium (EGaIn) are now the mostly used liquid metals in MEMS and microfluidic system because of their low toxicity, low viscosity, and low vapor pressure when compared to toxic liquid metal such as mercury. One of the important applications of EGaIn is the forming of electrodes in microfluidics systems $[9,10]$ which is now being commonly used as interdigital transducer (IDT) to generate acoustic waves. However, despite decades of extensive research, the dynamics of the filling of liquid metal into a microchannel is still poorly understood.

For common liquids such as water, we have demonstrated in a separate work that their flow into a closedend microchannel driven by a constant pressure [11] is a smooth and predictable process. Water enters the closedend microchannel continuously and smoothly. This process can be modeled using air diffusion through the porous polydimethylsiloxane (PDMS) wall. In this article, we show that the filling of EGaIn driven by a constant pressure into a closed microchannel is very different from the process of water. The flow of EGaIn experiences total stops and sudden jumps: the flow stays halted for a while, and the liquid column then jumps to a new posi- tion and stops again. The wordings 'jump' and 'stop' aim to reflect the completely different time scales of the observed flow dynamics (see Appendix B). To understand the physics underlining this interesting 'stop and jump' phenomenon, we propose here a mathematical model using gas diffusion and a random pressure component. The component is aimed to reflect the stochastic behaviour observed. Once a very limited number of free physical parameters associated with that surface interaction are adjusted, we found a good agreement and consistency between the experimental observations and the model predictions. We hypothesize that this interesting phenomenon should be mainly attributed to the formation of a breakable oxide layer when EGaIn is exposed to air [12]. Although this oxide layer provides good mechanical stability to the developed metallic structures in microchannels $[9,12,13]$, its formation and cracking while the liquid penetrates into the microchannel results in an unsteady flow $[13,14]$. While some innovative works focused on complex fluid behavior on porous media [15-18], to the best of our knowledge, little or no theoretical analysis has been made before to characterize the dynamics and unsteady flow behaviour of EGaIn in a closed-end porous microchannel, even though similar phenomena should be omnipresent in everyday technical applications such as the flow of molten metals in intricate molds (porous or not) or the flow of molten lava through cracks in the rocks. In this work we show the profound differences found with the unrestricted flow of a common liquid into a microchannel.

\section{FLOW OF LIQUID METAL IN CLOSED MICROCHANNELS}

Fig.1 shows a schematic sketch of the experimental setup. The PDMS microchannels were fabricated us- 
ing a SU8 mold (channel width $w=200 \mu \mathrm{m}$, average height $h \sim 47 \mu \mathrm{m}$ and channel length $L=2750 \mu \mathrm{m}$ ) using standard photo and soft lithography technique [19]. A pressure controller (Elveflow, OB1 MK3) was connected to the inlet to provide a constant driving pressure [11, 20]. EGaIn (495425,Sigma-Aldrich) was purchased from Sigma Aldrich and used directly. The PDMS device was mounted on an inverted microscope (Nikon TiE, Japan) and images were captured using a monochrome camera (Miro 3, Vision Research) at a rate of 10 frames per second (fps). The low frame rate was used to capture the entire filling process. We kept the outlet of the channel closed to allow a complete filling of EGaIn in all the microchannels. A complete filling will not be possible with an open channel. EGaIn filled the microchannels under different constant driving pressures $(\Delta P=1200$ and 1600 mbar, respectively) imposed at the inlet.

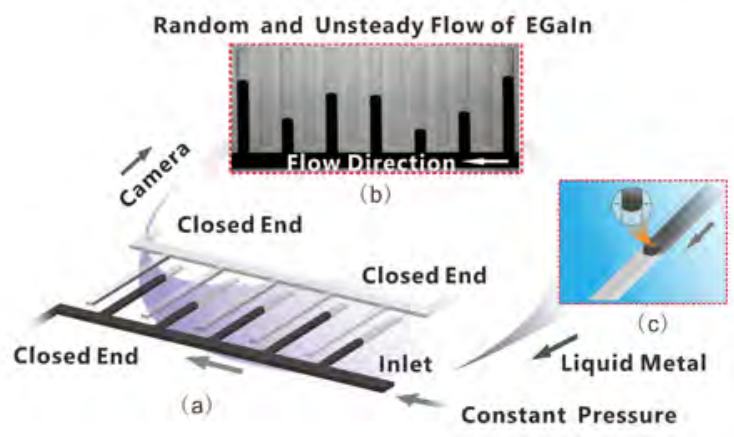

FIG. 1. Experimental setup. (a) microchannels. (b) image of the filling process . (c) 3D schematic of the PDMS channel.

We theoretically model the filling process of EGaIn in the closed-end microchannels with the following assumptions: (i) air can diffuse throughout the channel wall due to the (molecular) porosity of PDMS; and (ii) the diffusion time of momentum $\left(t_{\nu}=h w / \nu=9.4 \mathrm{~ms}\right.$, where $\nu=\mu / \rho$ is kinetic viscosity coefficient of the liquid) is much smaller than the diffusion of air into PDMS $\left(t_{D}=h w / D_{\text {gas }}=4.7 \mathrm{~s}\right.$, where $D_{\text {gas }}$ is the diffusion coefficient of air into PDMS, and $D_{\text {gas }} \sim 4.5 \times 10^{-9} \mathrm{~m}^{2} \mathrm{~s}^{-1}$ ). $\Delta P$ is the imposed pressure by the controller, where the absolute (total) gas pressure is $\Delta P+P_{a t m}$, and $P_{a t m}$ is the atmospheric pressure. We also assumed that the liquid has essentially the same pressure everywhere, and thus equal to the total pressure imposed by the pressure controller, $\Delta P+P_{a t m}$. We propose a dimensionless number $G=12 \mu D_{\text {gas }} L^{2} /\left[w h^{3}\left(P_{a t m}+\Delta P\right)\right]$ to measure the relative significance of viscous relaxation time along the channel for a given pressure to the time of diffusion into PDMS. In our experiment, the dimensionless number is estimated as $G \sim 10^{-4}$. This inevitably implies that gas diffusion dominates over the viscous flow effect. We emphasize that given the sizes of our channels, the current analysis is very different from the force analysis which is often used for the case of capillary filling, where one may find experimental studies with $G \sim 10^{6}$ [21]. In brief, our model captures the unsteady random flow behaviour of liquid metal in microchannels using subsequent steps of two metastable equilibrium states between (i) air escaping from the porous PDMS while the metal interface is "frozen" by the formation of that oxide layer, and (ii) the corresponding rapid spatial uptake of the liquid metal when that layer cracks due to the relative pressure build up at the metal phase with respect to the remaining air (given previous air escape or degassing), leading to new metal surface exposed to gas (oxygen).

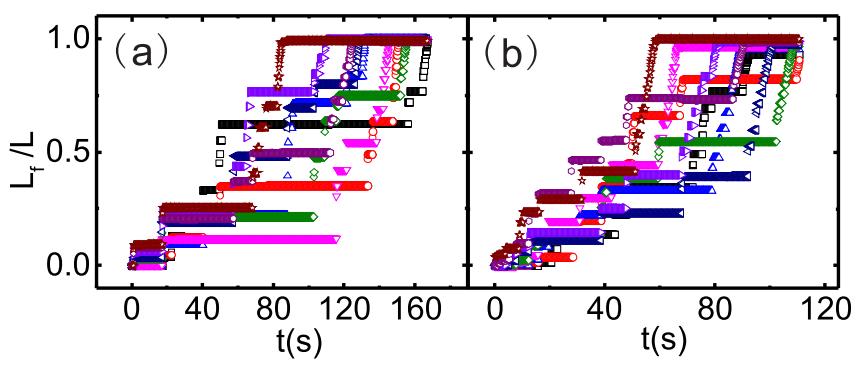

FIG. 2. Experimental measurements of the non-dimensional length of the EGaIn-filled part of each channel of the system as a function of time, under an imposed inlet pressure of (a) 1200 mbar and (b) 1600 mbar. Each color represent the filling process in each one of the nine microchannels considered. The plots illustrate the complexity of the global evolution due to the strong stochastic component present in the evolution of each individual microchannel.

Fig. 2 depicts the relative filling length $L_{f} / L$ of the random unsteady flow behaviour of EGaIn under constant pressures of $1200 \mathrm{mBar}$ and $1600 \mathrm{mBar}$, where $L_{f}$ is the filling length and $L$ is the total length of a microchannel. The data from 9 adjacent microchannels were captured and analysed using a customized Matlab image processing program. Fig. 2 clearly shows that the filling dynamics reflects a complex and intricate mechanism. For example, the average filling speed, the number of "step" periods, and the widths of each step period, are random and unpredictable. In this process, the trapped gas pressure in the unfilled part of the channel undergoes rapid increases due to the sudden advance of the liquid column followed by a relatively slow decrease by degassing. This occurs by diffusion of gas through the PDMS, which dominates the penetration of liquid in the channels. The diffusion process is described and modeled in Appendix A.

Besides, the advancement of EGaIn in the microchannel of constant section $h \times w$ is governed by the following equation:

$$
h w \frac{d \rho_{a} l}{d t}+l \int_{s_{l}}\left(0.79 \rho_{\mathrm{N}_{2}} v_{d, \mathrm{~N}_{2}}+0.21 \rho_{\mathrm{O}_{2}} v_{d, O_{2}}\right) \mathrm{d} s=0
$$

with the initial condition of $l(0)=L$, where $\rho_{a}=$ 
$0.79 \rho_{\mathrm{N}_{2}}+0.21 \rho_{\mathrm{O}_{2}}$ is the density of air trapped in the channel at pressure $P, l$ is the length of trapped air in the channel, and $s_{l}$ is the perimeter of the cross section of the channel. For a slender channel of constant cross section, one can assume an average gas diffusion $\bar{v}_{d}$ along the perimeter such that:

$$
\int_{s_{l}}\left(0.79 \rho_{\mathrm{N}_{2}} v_{d, \mathrm{~N}_{2}}+0.21 \rho_{\mathrm{O}_{2}} v_{d, O_{2}}\right) \mathrm{d} s=2(h+w) \rho_{a} \bar{v}_{d}
$$

taking as a good approximation $\rho_{a} \cong \rho_{\mathrm{N}_{2}} \cong \rho_{\mathrm{O}_{2}}$, where $\bar{v}_{d}=-\left.D_{\text {gas }} \frac{\partial Y}{\partial r}\right|_{r=D / 2}=-\left.2 \frac{D_{\text {gas }} \chi \Delta P}{D} \frac{\partial \psi}{\partial \eta}\right|_{\eta=1}$ is the mass outflow of the gas by Fick's first law . Here (Appendix A), we have defined the non-dimensional variables $\eta=2 r / D, \psi=\left(P_{P D M S}-P_{a t m}\right) / \Delta P$, and $Y=$ $\chi\left(P_{P D M S}-P_{a t m}\right)+Y_{0}=\chi \psi \Delta P+Y_{0}$ the mass fraction of air in PDMS. $\chi=0.79 \chi_{\mathrm{N}_{2}}+0.21 \chi_{\mathrm{O}_{2}}$ is the average permeability of PDMS to air. For simplicity, we define an equivalent cylindrical channel with diameter $D$ as $\pi D=2(h+w)$ (see Appendix A). Eq. (1) can be rewritten as

$$
\frac{h w}{2 \pi D_{\text {gas }} \chi \Delta P} \frac{1}{\rho_{a} l} \frac{d\left(\rho_{a} l\right)}{d t}-f(\tau)=0
$$

where $\left.\frac{\partial \psi}{\partial \eta}\right|_{\eta=1}=f(\tau)$ (Appendix A). We define $t_{0}=$ $D^{2} /\left(4 \cdot D_{\text {gas }}\right)=(h+w)^{2} /\left(\pi^{2} D_{\text {gas }}\right)$ as the characteristic diffusion time, and $\tau=t / t_{0}$. Solving Eq. (1) leads to

$$
\rho_{a} l=\rho_{a}(0) l(0) \cdot \exp \left(\alpha \int_{0}^{\tau} \mathrm{f}\left(\tau^{\prime}\right) \mathrm{d} \tau^{\prime}\right)
$$

where $\alpha=\frac{2(h+w)^{2}}{\pi h w} \chi \Delta P$. This solution governs the general liquid flow in porous PDMS microchannels, either smooth or step-like.

To resolve the present problem of the unsteady flow of EGaIn in microchannels, some considerations should be made:

- In general, the thermodynamic evolution of the gas in the channel is assumed to be isothermal, because the thermal relaxation time $t_{T} \sim(h w) / \alpha_{a}=7 \mathrm{~ms}$ (where $\alpha_{a}=1.9 \times 10^{-5} \mathrm{~m}^{2} / \mathrm{s}$ is the thermal diffusivity of air) is much smaller than $t_{0}\left(t_{0}=1.37 \mathrm{~s}\right.$. in our experiments). This would be the case when the liquid metal column is static during the random step-like unsteady flow behaviour of EGaIn in a microchannel.

- However, the sudden compressions taking place in the advance steps of the liquid is fast enough to invalidate this assumption.

- The actual length of the channel where gas is flowing into PDMS can be significantly different from $l(t)$, because the liquid metal does not easily wet the surface of the PDMS wall: the presence of a gas layer between the metal and the PDMS prevents wetting and is very often observed along the channel. Indeed, the same solid thin oxide crust that forms at the liquid surface in contact with the gas side of the channel is also formed along the whole channel surface, which allows gas trapping between the two solids (PDMS and oxide crust). This is observed from the experimental images and depicted in Fig.1(c). Thus, we have experimentally learned that the total length of the surface through which gas flows into the PDMS should be approximately equal to the total length $L$ of the channel.

- EGaIn forms a convex curvature when filling the microchannels. However, this curvature does not stay exactly the same throughout the process. In fact, as EGaIn fills the microchannel, we hypothesize that its surface becomes partially solidified by the heterogeneous build up of a metal oxide layer at the virgin metal interface as it forms [12]. This layer may momentarily anchor to the PDMS wall by friction or partial snagging. As a result, a momentary "stopping" behaviour is observed in this case.

Hence, the EGaIn flows in random "steps" as illustrated in figure 3 , showing the cycles of the pressure amplitude and its stochastic nature. Each $i$-step can be split in two parts with radically different time scales:

1. In the first part, the initial gas pressure in the unfilled (gas) side of the channel is $P_{1, i}$, and the length of the filled side $L_{f}$ initially remains constant during a time interval $\Delta t_{i}$. The characteristic time of this process is comparable to $t_{D} \sim h w / D_{\text {gas }}$, and the gas side with length $l_{i}=L-L_{f}$ degasses through the PDMS wall. Consequently, the pressure decreases until it reaches a (stochastic) level $P_{0, i+1}$ at which the EGaIn oxide layer breaks. This breakup finalizes the first part and exposes a virgin metal surface to the oxidizing gas.

2. The second part is characterized by a very fast advance of the liquid column in the channel, with a characteristic time comparable to $t_{2} \sim\left(\frac{\rho_{l} L^{2}}{\Delta P}\right)^{1 / 2} \sim$ $0.13 \times 10^{-3} \mathrm{~s} \ll t_{0} \sim t_{D}$. In our experiments, the liquid density is $\rho_{l}=6.25 \times 10^{3} \mathrm{Kg} / \mathrm{m}^{3}, L \sim 10^{-3} \mathrm{~m}$, $\Delta P \sim 10^{5}$ Pasc., and $t_{2} / t_{D} \sim 10^{-4}$. The viscosity of EGaIn is $\mu_{l}=2 \mathrm{mPa} \cdot \mathrm{s}$, which yields a characteristic viscous diffusion time in our channel $t_{v l}=$ $\rho_{l} h w / \mu_{l}=0.03 \mathrm{~s} \gg t_{2}$. Besides, thermal conductivity of PDMS is approximately $K_{s}=0.15 \mathrm{~W} /(\mathrm{m}$ $\mathrm{K})$, its density $\rho_{s}=970 \mathrm{~kg} / \mathrm{m}^{3}$, and its specific heat $\left.C_{p, s}\right)=1460 \mathrm{~J} /\left(\mathrm{kg}^{\circ} \mathrm{K}\right)$, which yields a thermal diffusion coefficient $\beta_{s}=K_{s} /\left(\rho_{s} C_{p, s}\right) \simeq 10^{-7}$ 
$\mathrm{m}^{2} / \mathrm{s}$ and a characteristic time for thermal diffusion in the PDMS $t_{s}=h w / \beta_{s}=0.1 \mathrm{~s} \gg t_{2}$. Then, we assume that the rapid advance of the compression step takes place adiabatically and with no diffusion of mass or heat through the PDMS. The length of the unfilled side suddenly decreases from $l_{i}$, the gas becomes compressed, the column rapidly decelerates, and the quickly oxidized liquid metal front snags again onto the PDMS surface. At this point, the column stops at a new length $L_{f}=L-l_{i+1}$, where $l_{i+1}<l_{i}$ is the new length of the unfilled side, reaching a pressure $P_{1, i+1}$. Naturally, the difference $P_{1, i+1}-P_{0, i+1}$ should be comparable to the Laplace pressure of the virgin liquid metal, but it should be randomly distributed around that pressure to reflect the random interface curvature resulting from the irregular breakup and rebuilt of the oxide metal layer, and its irregular snagging to the PDMS surface.This finalizes the second part of step $i$ and initiates the next step $i+1$.

To capture the above description, solution (3) should be properly adopted in a step-wise fashion. First, for the degassing part of each $i$-step under a constant length $l_{i}$, using equation (4), the ratio of initial to final pressures $P_{0, i+1} / P_{1, i}$ can be expressed as

$$
\frac{P_{0, i+1}}{P_{1, i}}=\exp \left(\alpha_{i} \int_{\tau_{i-1}}^{\tau_{i}} f(\tau) d \tau\right)
$$

where $\alpha_{i}=\frac{2(h+w)^{2}}{\pi h w\left(l_{i} / l_{0}\right)}\left(0.79 \chi_{\mathrm{N}_{2}}+0.21 \chi_{\mathrm{O}_{2}}\right) \Delta P$. Assuming an average value of $\langle f(\tau)\rangle=B<0$ for our particular device, independent of the operating conditions, one can express

$$
\frac{P_{0, i+1}}{P_{1, i}}=\exp \left(\alpha_{i} B \Delta \tau_{i}\right)
$$

Constant $B(<0)$ shall be a fitting parameter to be calculated from experiments.

Secondly, according to the rapid adiabatic process, the gas pressures $P_{0, i+1}$ and $P_{1, i+1}$ just before and after the sudden compression (advancement), respectively, are related to the lengths at each $i$-step as:

$$
\left(\frac{l_{i+1}}{l_{i}}\right)^{\gamma}=\frac{P_{0, i+1}}{P_{1, i+1}}
$$

where $\gamma=1.4$ is the adiabatic index for air. Here, the pressure difference between the gas and the liquid should be a function of its surface tension, responsible to withstand the curvature of the liquid metal front. That curvature is maximum (minimum) when the gas pressure reaches $P_{0, i+1}\left(P_{1, i+1}\right)$.

Thus, one can write the following approximate expression for the degassing part of step $i$ :

$$
\frac{P_{0, i+1}}{P_{1, i}}=\exp \left(\alpha_{i} B \Delta \tau_{i}\right) \simeq \frac{P_{a t m}+\Delta P-P_{L, i, d}}{P_{a t m}+\Delta P+P_{L, i-1, c}}
$$

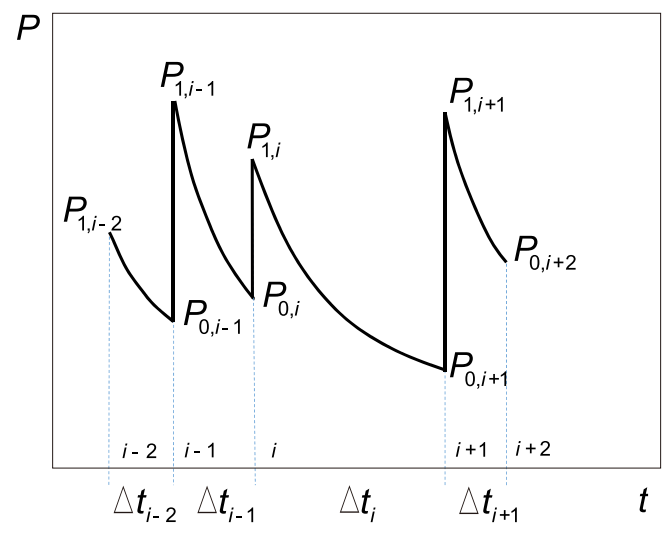

FIG. 3. Illustration of the random sudden compression and slow degassing processes taking place during the penetration of the EGaIn in the PDMS channel.

where $P_{L} \sim \sigma / h$ is the static Laplace pressure at the interface between the gas and EGaIn, and $\sigma=0.624 \mathrm{~N} / \mathrm{m}$ is the surface tension of the virgin liquid EGaIn [9]. We have distinguished the random Laplace-related pressure at the end of the degassing $i$-process $P_{L, i, d}$ from the random one after sudden compression of previous step $P_{L, i-1, c}$. In average, the Laplace overpressures are much smaller than the applied pressure, and therefore one may approximately write equation (6), after the appropriate linearization:

$$
\exp \left(\alpha_{i} B \Delta \tau_{i}\right) \simeq 1-\frac{P_{L, i, d}+P_{L, i-1, c}}{P_{a t m}+\Delta P} \equiv 1-X_{i}
$$

Similarly, for the compression part of step $i$ (equation 7 ), one can write:

$$
\frac{P_{0, i+1}}{P_{1, i+1}}=\left(\frac{l_{i+1}}{l_{i}}\right)^{\gamma} \simeq 1+\frac{P_{L, i, c}+P_{L, i, d}}{P_{a t m}+\Delta P} \equiv 1+X_{i}^{\prime}
$$

The combined statistical variables $X_{i}$ and $X_{i}^{\prime}$ can be measured from experiments for different applied pressures, using the measured values of the successive lengths $l_{i}$ taken from experiments (videos) and equation (7). A few fundamental considerations should be made here:

1. Even though $P_{L, i, c}$ and $P_{L, i, d}$ may have different distributions, the measured distributions of $X_{i}$ and $X_{i}^{\prime}$ should approximately match because $P_{L, i, c}$ and $P_{L, i, d}$ are intermixed (riffled) in $X_{i}$ and $X_{i}^{\prime}$. In our experiments, we will match both mean values $\left\langle X_{i}\right\rangle$ and $\left\langle X_{i}^{\prime}\right\rangle$ to calculate the fitting constant $B$.

2. The experimental measurements of $\tau_{i}$ and $l_{i}$ should be carefully filtered to eliminate sources of small deviations due to noise (vibrations, etc.)

3. Degassing steps leading to very large leaps in time may yield overpressures which are not small compared to $P+\Delta P$. Given that those events are rare, one can suppress them from the statistical analysis. 
4. In this analysis, we have neglected any possible dynamical coupling among the different channels.However, we are conscious that this coupling exists in reality at certain level, which produces a further stochastic restriction in the mathematical behavior of the tails of the probability density functions; yet, these restrictions do not affect the averages of the involved variables nor the validity of present analysis. This is subsequently discussed.

Figure 4 shows the statistical distributions of both $X_{i}$ and $X_{i}^{\prime}$. Using a fitting constant $B=-9$, both distributions can be relatively well approximated (considering the significant experimental uncertainties and the limited number of experiments available) by a log-normal distribution with mean $\mu=-4$ and standard deviation $\xi=2$. This yields average values $\langle X\rangle=\left\langle X^{\prime}\right\rangle=0.018$.

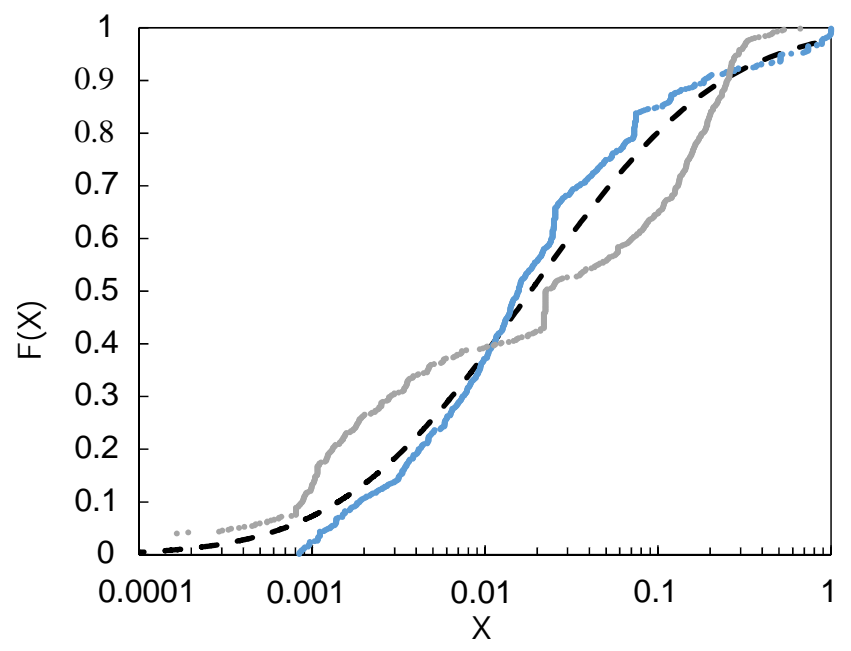

FIG. 4. Statistical cumulative distributions of both $X_{i}$ (blue line) and $X_{i}^{\prime}$ (gray line). Black dashed line is a lognormal cumulative distribution function $F(X)$ with mean $\mu=-4$ and standard deviation $\xi=2$.

In summary, the following fitting parameters are used in our model:

1. The value of $B$, which physically reflects the (nondimensional) tendency of the gas to escape through the PDMS, is uniquely determined by the device geometry and its material (PDMS).

2. The parameters of the statistical distribution that fits the experimental distributions of random Laplace pressures, which reflect the randomness of the process.

Fig. 5 presents a selected simulation based on the results from our model. We observe that the model finely captures the compression and degassing steps, and the whole physics of the liquid metal filling process. A perfect fitting between the experimental data and the model is

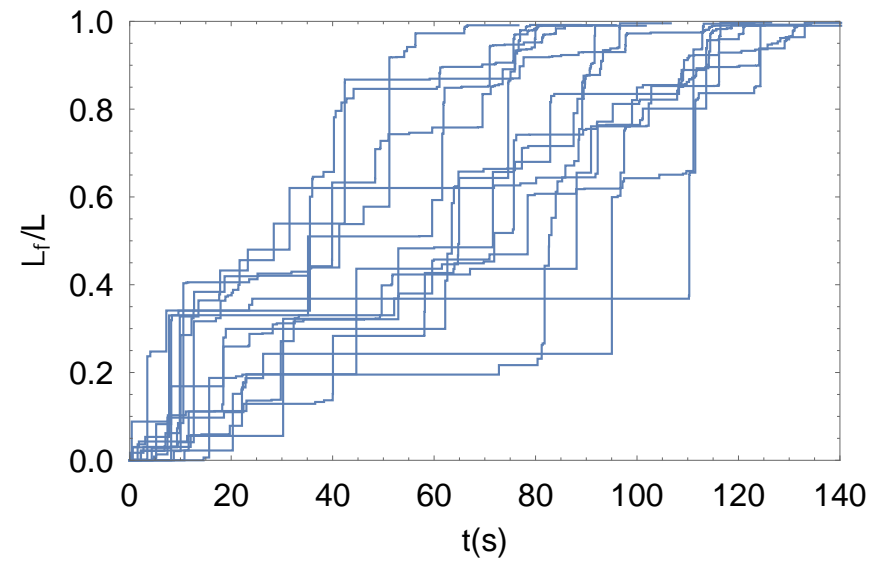

FIG. 5. Simulation of EGaIn filling process under an imposed inlet pressure of 1600 mbar, 15 simulated runs (or independent channels).

virtually impossible due to the unpredictable randomness of the liquid metal flowing in each individual microchannel. However, our model provides accurate predictions using the ensemble average method. An important observation here is that the simulated filling represents the behaviour of independent channels. However, our particular system comprises 9 relatively close channels arranged in parallel and all them connected to a common feeding large channel (see Fig. 1): this provokes a measurable interaction among the different channels reflected in a phenomenon of synchronism that will be studied in further works. In particular, we can advance that if there was not synchronism at all, dividing the whole filling time interval in small sections (for example, of $0.1 \mathrm{~s}$ each) the coincidence of leaps within a certain time section should follow a Poisson distribution. However, this is not the case for our system: the probability of finding simultaneous leaps in the same time section becomes orders of magnitude larger than that predicted by a Poisson distribution. Although this would not affect the average and standard deviations of the statistical distributions shown in Fig. 4, their shapes (i.e. higher order moments of the distributions) could be significantly affected, as observed. Indeed, one may observe a non-natural shape of the distribution of $X_{i}^{\prime}$ around its median. All this is why the experimental evolution shown in Fig. 2 exhibits much less dispersion than a simulation with a large number of runs; however, the average of those runs always yields the same asymptotic result, naturally.

The arithmetic average of the experimental data can be compared to the average results from the model. The fitting between both experimental results and model with a single fitting constant and a fitting experimental statistical distribution is shown in Fig. 6. The simulated results come from the arithmetic average of the model results of 100 runs. Even though we use experimental data for only 9 fingers, there is very good consistency be- 
tween the simulated results and the experimental ones, both for imposed inlet pressure of 1200 mbar and 1600 mbar. Thus, our model and the corresponding fitting parameters capture the physics and explain satisfactorily the unsteady random flow of EGaIn in a microchannel of a porous matrix like PDMS.

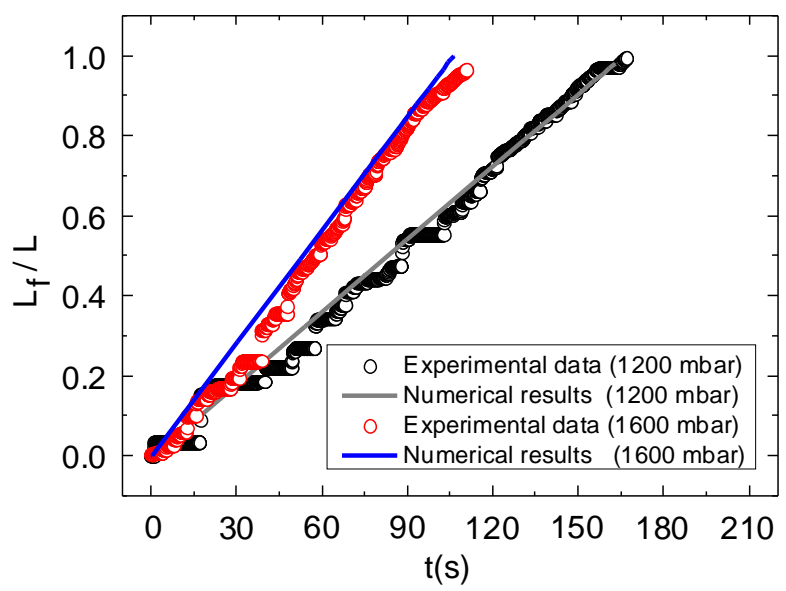

FIG. 6. Average filling length of liquid metal in all captured fingers under the imposed inlet pressure of 1200 mbar (left) and 1600 mbar (right), respectively. The open dots come from experimental data, and the solid lines denote the numerical results.

In conclusion, the random unsteady flow behaviour of EGaIn in porous microchannels under a constant driving pressure is theoretically investigated and experimentally validated. Complete filling of the porous microchannels can be realized by imposing a constant pressure at the inlet. We observed a random "stopping" and "jumping" phenomenon, which is attributed to the formation of a transient oxide layer that subsequently cracks and exhibits a new liquid surface, allowing the liquid column to penetrate into the porous microchannel. That new surface is oxidized again and eventually snags on the PDMS surface after a transient time, stopping the advance of the column. A theoretical model based on the gas diffusion and random surface tension overpressures is developed to describe this phenomenon. The physical properties and experimental data are well captured and described by our model. Our work offers the basic understanding of the unsteady random flow of liquid metals in porous microchannels prefilled with an oxidizing gas such as air.

\section{ACKNOWLEDGEMENTS}

This work was partially performed at the Queensland Node of the Australian National Fabrication Facility, a company established under the National Collaborative Research Infrastructure Strategy to provide nano and micro-fabrication facilities for Australia's researchers. A. M. Gañán-Calvo acknowledges support from the Min- isterio de Economía y Competitividad through project DPI2016-78887-C3-1R. S. H. Tan and N.T. Nguyen gratefully acknowledges the support of the Australian Research Council (ARC) Discovery Early Career Researcher Award (DECRA) (DE170100600), Griffith UniversitySimon Fraser University and Griffith University-Peking University collaboration grant. W. Guo and H.-D. Xi gratefully acknowledge the support by the National Natural Science Foundation of China (through grant nos. 11472094, 11772259, and U1613227), and the 111 project of China (B17037).

\section{APPENDIX}

\section{Gas diffusion model in the PDMS matrix}

Here, we propose an equivalent cylindrical channel, with a diameter of $\mathrm{D}$, to approximate the rectangular microchannels. This equivalence is based on the same perimeters of two different geometries, which gives $2(h+w)=\pi D$ or $D=\frac{2(h+w)}{\pi}$. In this way, the conversion provides simplicity when we model the system in polar coordinates. We assumed that the air in the PDMS channel consists of two main components: nitrogen and oxygen. Then we investigate the filling process in the channel domain $\left(0<r<\frac{D}{2}\right)$ and the gas diffusion in the PDMS domain $\left(r>\frac{D}{2}\right)$. In the following, $P_{P D M S}(r)$, $Y(r)$, and $C(r)$ denote the pressure of air in the PDMS domain, the mass fraction of gas in the PDMS domain, and the volume fraction of gas in PDMS, respectively. Sadrzadhel et al [22] reported that nitrogen and oxygen have similar permeability in PDMS, which can be expressed as

$$
\begin{aligned}
& \frac{\partial C_{\mathrm{N}_{2}}}{\partial P}=9.35 \times 10^{-7} \mathrm{~Pa}^{-1} \\
& \frac{\partial C_{\mathrm{O}_{2}}}{\partial P}=1.87 \times 10^{-6} \mathrm{~Pa}^{-1}
\end{aligned}
$$

Equation (11) leads to the permeabilities written in terms of the mass fractions of each gas component of air (the form used in this work for the gas permeabilities):

$$
\left\{\begin{array}{l}
\chi_{\mathrm{N}_{2}} \equiv \frac{\partial Y_{\mathrm{N}_{2}}}{\partial P}=\frac{\rho_{\mathrm{N}_{2}}}{\rho_{\mathrm{PDMS}}} \frac{\partial C_{\mathrm{N}_{2}}}{\partial P}=1.1 \times 10^{-9} \mathrm{~Pa}^{-1} \\
\chi_{\mathrm{O}_{2}} \equiv \frac{\partial Y_{\mathrm{O}_{2}}}{\partial P}=\frac{\rho_{\mathrm{O}_{2}}}{\rho_{\mathrm{PDMS}}} \frac{\partial C_{\mathrm{O}_{2}}}{\partial P}=2.5 \times 10^{-9} \mathrm{~Pa}^{-1}
\end{array}\right.
$$

For a given pressure $P$ in the unfilled part of the channel (gas side), the mass fraction of the gas, either nitrogen or oxygen, in the PDMS domain can be calculated from (12) as

$$
Y-Y_{0}=\chi\left(P_{P D M S}-P_{a t m}\right)
$$

where $P_{\mathrm{PDMS}}$ is the gas pressure in the PDMS, and $\chi=$ $0.79 \chi_{\mathrm{N}_{2}}+0.21 \chi_{\mathrm{O}_{2}}$ is the average permeability of PDMS 
to air. According to Fick's second law, the diffusion of gas into PDMS is governed by

$$
\frac{\partial Y}{\partial t}=D_{g a s} \frac{1}{r} \frac{\partial}{\partial r}\left(r \frac{\partial Y}{\partial r}\right)
$$

In a smooth filling process, such as that of water or ethanol, the air trapped is pushed by the liquid column at a constant pressure along the process (i.e. there is no "stopping" or "jumping"). Then, the boundary conditions (B.C.) in this case are:

$$
\left\{\begin{array}{l}
Y(r=D / 2, t)=Y\left(P_{P D M S}=P\right) \\
Y(r \gg D / 2, t)=Y\left(P_{P D M S}=P_{a t m}\right) \equiv Y_{0} \\
\frac{\partial Y}{\partial t}(r=D / 2, t)=\chi \frac{d P}{d t}
\end{array}\right.
$$

Here $D_{\text {gas }}$ is the diffusion coefficient of the gas in PDMS, which is almost the same for both $\mathrm{N}_{2}$ and $\mathrm{O}_{2}: D_{\text {gas }} \sim 4.5 \times 10^{-9} \mathrm{~m}^{2} \mathrm{~s}^{-1}[22]$. We define the non-dimensional variables $\eta=2 r / D, \tau=t / t_{0}, \psi=$ $\left(P_{P D M S}-P_{a t m}\right) / \Delta P$ and $Y=\chi\left(P_{P D M S}-P_{a t m}\right)+$ $Y_{0}=\chi \psi \Delta P+Y_{0}$, where $t_{0}=D^{2} /\left(4 \cdot D_{\text {gas }}\right)=$ $(h+w)^{2} /\left(\pi^{2} D_{\text {gas }}\right)$ denotes the characteristic diffusion time. Eq. (14) can be nondimensionalized as

$$
\frac{\partial \psi}{\partial \tau}=\frac{1}{\eta} \frac{\partial}{\partial \eta}\left(\eta \frac{\partial \psi}{\partial \eta}\right)
$$

The linear nature of Eq. (15) allows its analytical solution with B.C. to be decomposed as the sum of particular solutions:

$$
\psi(\eta, \tau)=\sum_{i} \psi_{i}(\eta, \tau)
$$

where $\psi_{i}(\eta, \tau)$ denotes different solutions satisfying Eq. (15) with generic B.C. . Here, we consider three of those particular solutions. First, we introduce the simplest one,

$$
\psi_{0}(\eta, \tau)=A_{0}\left(\tau+\frac{\eta^{2}}{4}\right)+B \ln \eta+A_{1}
$$

that fulfil certain but not the whole set of B.C. To gain refinement with additional solutions, we can introduce a self-similar variable $\xi=\eta^{2} /(4 \tau)$ that leads to

$$
\left\{\begin{array}{l}
\frac{\partial}{\partial \tau}=\frac{d}{d \xi} \frac{\partial \xi}{\partial \tau}=-\frac{\eta}{4 \tau^{2}} \frac{d}{d \xi} \\
\frac{\partial}{\partial \eta}=\frac{d}{d \xi} \frac{\partial \xi}{\partial \eta}=\frac{\eta}{2 \tau} \frac{d}{d \xi}
\end{array}\right.
$$

and transforms Eq. (15) to an ordinary equation

$$
\left(\xi \psi^{\prime}\right)^{\prime}+\xi \psi^{\prime}=0
$$

with B.C.:

$$
\left\{\begin{array}{l}
\psi(\eta=1, \tau)=1 \\
\psi(\eta \gg 1, \tau)=0 \\
\frac{\partial \psi}{\partial \tau}(\eta=1, \tau)=t_{0} \frac{1}{\Delta P} \frac{d\left(P-P_{a t m}\right)}{d t}
\end{array}\right.
$$

A generic analytical solution to Eq. (18) is $\varphi=C$. $\exp (-\xi)$, where $\varphi=\xi \psi^{\prime}$. Thus we have

$$
\psi_{1}(\xi)=C \cdot \operatorname{Ei}(-\xi)
$$

where $E i(x)$ is the exponential integral function and expressed as $E i(x)=-\int_{-x}^{\infty} \frac{e^{-t}}{t} d t$. Again, this solution does not completely resolve the whole set of B.C. Finally, using the method of separation of variables, Eq. (15) can also be solved as

$$
\psi_{2}(\eta, \tau)=\int_{-\infty}^{\infty} A(\lambda) \cdot \exp (-\lambda \tau) \cdot K_{0}(\lambda \eta) d \lambda
$$

where $K_{0}$ is the modified Bessel function of order 0 , and $A(\lambda)$ reflects the solution to the set of B.C. not fulfilled by $\psi_{0}$ or $\psi_{1}$. In summary, the analytical solution to Eq. (15) can be finally written as the sum of (17), (19), and (20):

$$
\psi(\eta, \tau)=\psi_{0}(\eta, \tau)+\psi_{1}(\xi)+\psi_{2}(\eta, \tau)+\cdots
$$

where $A_{0}, A_{1}, A(\lambda), B$ and other coefficients are solved to meet the initial and B.C. conditions of gas diffusion in PDMS. In a smooth filling process, the pressure in the unfilled (gas) part of the channel is constant, which means $g(\tau)=0$ and results in:

$$
\begin{aligned}
f(\tau)= & B+\frac{C}{\tau} \cdot(1+2 \tau) \cdot \exp \left(-\frac{1}{4 \tau}\right)+ \\
& \int_{-\infty}^{\infty} \lambda A(\lambda) \exp (-\lambda \tau)\left[K_{0}(\lambda)-K_{1}(\lambda)\right] d \lambda
\end{aligned}
$$

where $K_{0}$ is the modified Bessel function of order 1. However, in the case of a step-like evolution (liquid EGaIn) the gas pressure is not constant in each degassing step at constant length. The B.C. are in this case.:

$$
\begin{aligned}
& \left.\frac{\partial Y}{\partial r}\right|_{r=D / 2}=\left.\frac{\pi \chi \Delta P}{h+w} \frac{\partial \psi}{\partial \eta}\right|_{\eta=1}=\frac{\pi \chi \Delta P}{h+w} \cdot f(\tau) \\
& \left.\frac{\partial Y}{\partial \tau}\right|_{r=D / 2}=\left.\frac{\chi \Delta P}{t_{0}} \frac{\partial \psi}{\partial \tau}\right|_{\eta=1}=\frac{\chi \Delta P}{t_{0}} \cdot g(\tau)
\end{aligned}
$$

where $f(\tau)=\left.\frac{\partial \psi}{\partial \eta}\right|_{\eta=1}$ and $g(\tau)=\left.\frac{\partial \psi}{\partial \tau}\right|_{\eta=1}$, respectively. A general solution of this problem cannot be analytically obtained, and should be numerically resolved for each particular configuration. However, some simplifying hypotheses can be made on $f(\tau)$ such that it can be approximately obtained from experiments for each given configuration.

In summary, $f(\tau)$ is the key factor which determines the degassing process through the PDMS that leads to the advancement of the liquid column.

\section{Video of liquid metal filling}

A video showing the filling of the liquid metal(EGaIn,Sigma Aldrich) with inlet pressure at $1600 \mathrm{mBar}$. The video captured the stepped motion of the liquid metal. This is attributed to the constant formation and breaking of the oxide layer formed with exposure to air. This video was captured at $10 \mathrm{fps}$ and played at a rate of $50 \mathrm{fps}$. 
* Email for correspondance: amgc@us.es;

† Email for correspondance: sayhwa.tan@griffith.edu.au

[1] J. Taber et al., Society of Petroleum Engineers Journal 9, 3 (1969).

[2] M. Bruschke and S. G. Advani, Polymer composites 11, 398 (1990).

[3] I. D. Joshipura, H. R. Ayers, C. Majidi, and M. D. Dickey, Journal of Materials Chemistry C 3, 3834 (2015).

[4] P. Sen and C.-J. C. Kim, IEEE Transactions on Industrial Electronics 56, 1314 (2009).

[5] S.-Y. Tang, K. Khoshmanesh, V. Sivan, P. Petersen, A. P. O'Mullane, D. Abbott, A. Mitchell, and K. Kalantarzadeh, Proceedings of the National Academy of Sciences 111, 3304 (2014).

[6] A. Traille, S. Bouaziz, S. Pinon, P. Pons, H. Aubert, A. Boukabache, and M. Tentzeris, in Microwave Conference (EuMC), 2011 41st European (IEEE, 2011) pp. $45-48$.

[7] J.-H. So and M. D. Dickey, Lab on a Chip 11, 905 (2011).

[8] D. Rodrigo, L. Jofre, and B. A. Cetiner, IEEE transactions on antennas and propagation 60, 1796 (2012).

[9] M. D. Dickey, R. C. Chiechi, R. J. Larsen, E. A. Weiss, D. A. Weitz, and G. M. Whitesides, Adv. Funct. Mater. 18, 1097 (2008).

[10] H.-D. Xi, W. Guo, M. Leniart, Z. Z. Chong, and S. H. Tan, Lab on a Chip 16, 2982 (2016).

[11] W. Guo, A. Teo, A. M. Gañán-Calvo, N.-T. Nguyen, C. Song, H.-D. Xi, and S. H. Tan, submitted (under review).
[12] K. Khoshmanesh, S.-Y. Tang, J. Y. Zhu, S. Schaefer, A. Mitchell, K. Kalantar-zadeh, and M. D. Dickey, Lab Chip (2017).

[13] S.-Y. Tang, K. Khoshmanesh, V. Sivan, P. Petersen, A. P. O'Mullane, D. Abbott, A. Mitchell, and K. Kalantarzadeh, Proc. Natl. Acad. Sci. U S A. 111, 3304 (2014).

[14] P. Nikola, Z. Qing, and J. David, J. Micromechanics Microengineering 22, 097001 (2012).

[15] M. Trojer, M. L. Szulczewski, and R. Juanes, Phys. Rev. Applied 3, 054008 (2015).

[16] M. B. Pinson, E. Masoero, P. A. Bonnaud, H. Manzano, Q. Ji, S. Yip, J. J. Thomas, M. Z. Bazant, K. J. Van Vliet, and H. M. Jennings, Phys. Rev. Applied 3, 064009 (2015).

[17] M. T. Bishop, K. H. Langley, and F. E. Karasz, Phys. Rev. Lett. 57, 1741 (1986).

[18] A. H. Thompson, A. J. Katz, and R. A. Raschke, Phys. Rev. Lett. 58, 29 (1987).

[19] S. H. Tan, N.-T. Nguyen, Y. C. Chua, and T. G. Kang, Biomicrofluidics 4, 032204 (2010).

[20] A. J. Teo, K.-H. H. Li, N.-T. Nguyen, W. Guo, N. Heere, H.-D. Xi, C.-W. Tsao, W. Li, and S. H. Tan, Analytical chemistry 89, 4387 (2017).

[21] V. N. Phan, N.-T. Nguyen, C. Yang, P. Joseph, L. Djeghlaf, D. Bourrier, and A.-M. Gue, Langmuir 26, 13251 (2010).

[22] M. Sadrzadeh, K. Shahidi, and T. Mohammadi, J. Appl. Polym. Sci. 117, 33 (2010).

[23] Z. Ma, A. J. Teo, S. H. Tan, Y. Ai, and N.-T. Nguyen, Micromachines 7, 216 (2016). 